\title{
Revisión
}

\section{Fiebre amarilla: un peligro latente}

Marvin Yglesias-Rosales ', Andrea Rodríguez-González ², Manuel Rojas-Montero ${ }^{3}$

\section{Resumen}

La fiebre amarilla es una zoonosis, aguda, febril que se encuentra catalogada como una fiebre hemorrágica potencialmente mortal. Es causada por un arbovirus y transmitida por un artrópodo del cual se conocen dos modalidades epidemiológicas, con un área endémica que corresponde a la mayoría de América del Sur y parte del continente africano. En Costa Rica la última epidemia de fiebre amarilla ocurrió a principios de los años cincuenta.

La fisiopatología de la fiebre amarilla no es bien conocida. La mayoría de las infecciones son sintomáticas, y tienen una alta mortalidad que varia según la epidemia. El diagnóstico se confirma de forma definitiva con la serología y aunque éste examen no se realiza en el país, las muestras de los casos sospechosos son enviadas a laboratorios panameños.

Con base en su gran mortalidad y a la ausencia de un tratamiento específico, es obvio que el camino a seguir en el manejo de la fiebre amarilla es la prevención mediante las vacunaciones masivas en las áreas endémicas y grupos de riesgo, así como el control del vector.

La fiebre amarilla sigue siendo una entidad que afecta considerablemente la salud pública en varios países del mundo; Costa Rica reúne un conjunto de condiciones que facilitarían su aparición y propagación, por lo que es una patología cuyas características convendría mantener en mente.

Descriptores: Arbovirus, fiebre amarilla, Costa Rica, zoonosis, fiebre hemorrágica

Key Words: Arbovirus, yellow fever, zoonosis, haemorragic fever.

Recibido: 18 de marzo de 2005

Aceptado: 31 de mayo de 2005

- Clínica Marcial Fallas.

2. Area de Salud Alajuela Oeste. Caja Costarricense de Seguro Social.

3. Servicio de Infectología del Hospital San Juan de Dios. Caja Costarricense de Seguro Social.

Abreviaturas: ARN, Acido Ribonucléico; ELISA, EnzymeLinked Immunosorbent Assay; IgM, Inmunoglobulina M

Correspondencia: Marvin Yglesias Rosales, Apto: 3052070 San José, Costa Rica; Fax: 253-5I-16.

ISSN 0001-6002/2005/47/3/118-125 Acta Médica Costarricense, (2005 Colegio de Médicos y Cirujanos
Ha pasado medio siglo desde el último caso registrado de fiebre amarilla en el país, pero aún así la amenaza de un nuevo brote persiste todavía en nuestros días. La fiebre amarilla es una zoonosis, aguda, febril y potencialmente mortal, causada por un arbovirus y transmitida por un artrópodo, se cataloga como una fiebre hemorrágica.

El motivo de éste artículo es promover la curiosidad por esta enfermedad, para que sea revisada y de ser necesario, contemplada como uno de los diagnósticos diferenciales al abordar un paciente febril.

\section{Historia}

La descripción más temprana de la enfermedad documentada se encuentra en el PopolVuh1, libro sagrado de los mayas, donde se relata la epidemia de una enfermedad llamada Xekik (vómito de sangre) ocurrida entre los años 1480 - 1485, doce años antes de la llegada de los españoles a América. 
Algunos autores asumen que los huevos de Aedes aegypti fueron importados en las carabelas de los españoles, lo que significó la introducción de un nuevo vector al ciclo de la enfermedad y que se manifestó como devastadores brotes de fiebre amarilla en los asentamientos españoles ${ }^{1}$.

Inicialmente la posibilidad de que la fiebre amarilla fuera trasmitida por un mosquito fue mencionado por el médico cubano Carlos Finlay en 1881, pero no pudo ser comprobado hasta 1900 gracias a los trabajos de Walter Reed y la comisión de fiebre amarilla del ejército estadounidense en Cuba ${ }^{2,3}$. Ellos controlaron la epidemia de fiebre amarilla que azotaba la Habana en tan solo 3 meses, implementando medidas para controlar el vector.

La última epidemia de fiebre amarilla en Costa Rica ocurrió a principios de los años cincuentas. En 1949 una epidemia de fiebre amarilla que se había iniciado en América del Sur se extendió hasta Panamá, posteriormente la epidemia se propagó a lo largo de las regiones montañosas lluviosas de la costa norte de Panamá e invadió Costa Rica a lo largo de la vertiente montañosa del Atlántico. En abril de 1951 se registraban las primeras muertes en territorio costarricense. El último caso de fiebre amarilla registrado en Costa Rica fue en 1953 en Río Cuarto de Grecia ${ }^{4}$.

\section{El virus}

Los Flaviviridae son arbovirus y su familia consiste de tres géneros: Flavivirus, Pestivirus y Hepacivirus. Dentro de los flavivirus se encuentran clasificadas enfermedades como el dengue, la fiebre del Nilo, la encefalitis de San Luis y la fiebre amarilla, entre otros.

El virus de la fiebre amarilla tiene un genoma ARN, monocatenario, positivo, cuyo material genético codifica un total de 10 proteínas, 3 estructurales y 7 no estructurales. Solo existe un serotipo ${ }^{5}$.

Entre las proteínas estructurales, la proteína E es la que se encuentra en mayor cantidad en la superficie y es probablemente integral en la unión a receptores y fusión con la membrana de la célula hospedera. La proteína $M$ es esencial para la maduración de proteínas virales inmaduras a formas infecciosas y la proteína $\mathrm{C}$ ayuda a construir las nucleocápsides ${ }^{6}$.

\section{Epidemiología}

Se conocen dos modalidades epidemiológicas fundamentales de la fiebre amarilla, la urbana y la selvática. En el ciclo urbano el virus se transmite de un hombre a otro por medio de la picadura de un mosquito Aedes aegypti, además se han implicado otros mosquitos como el Aedes albopictus ${ }^{7}$. En cuanto al ciclo selvático se caracteriza por la transmisión del virus de un mono a otro, por medio de mosquitos del género Haemagogus, el hombre se infecta accidentalmente al recibir la picadura de un Haemagogus a su vez infectado ${ }^{8-10}$.

En la actualidad se consideran endémicas las áreas ubicadas entre el paralelo 12 de latitud norte y el paralelo 12 de latitud sur tanto en el continente africano (África SubSahariana) como americano (Perú, Bolivia, Venezuela, Colombia y Brasil); pero la enzoótica no se encuentra en permanente actividad en todas partes, sino que presenta un comportamiento endemo-epidémico, presentando brotes cada cierto número de años ${ }^{3,11}$. (Figura 1)

El ciclo selvático se lleva a cabo en los grandes bosques, sobre todo en la cuenca hidrográfica del Amazonas y el Orinoco. El hombre adquiere la fiebre amarilla en algún punto de la periferia de éstas zonas boscosas, la transmisión se ve amplificada durante las épocas lluviosas debido a que el número de vectores aumenta y éstos se desplazan más allá de los límites de las áreas boscosas. Las personas que con mayor frecuencia se ven afectadas son los hombres de 10-40 años, por ser el grupo más activo en las labores agrícolas ${ }^{10}$. Sólo es necesaria una persona infectada para iniciar una epidemia en una zona con vectores domésticos ${ }^{12}$, pero debido a que los seres humanos no son buenos huéspedes para el virus de la fiebre amarilla, las concentraciones del virus en sangre son relativamente bajas por lo cual la infección de mosquitos a partir de seres humanos no es muy eficiente ${ }^{13}$.

Es esencial que las autoridades de salud sepan en donde se encuentra el virus activo y cual es el riesgo de su transmisión al hombre, hasta el momento las especies animales que han servido en forma más adecuada para apreciar el comportamiento de la enzootia selvática son los que pertenecen al orden de los primates. Es sabido que algunos

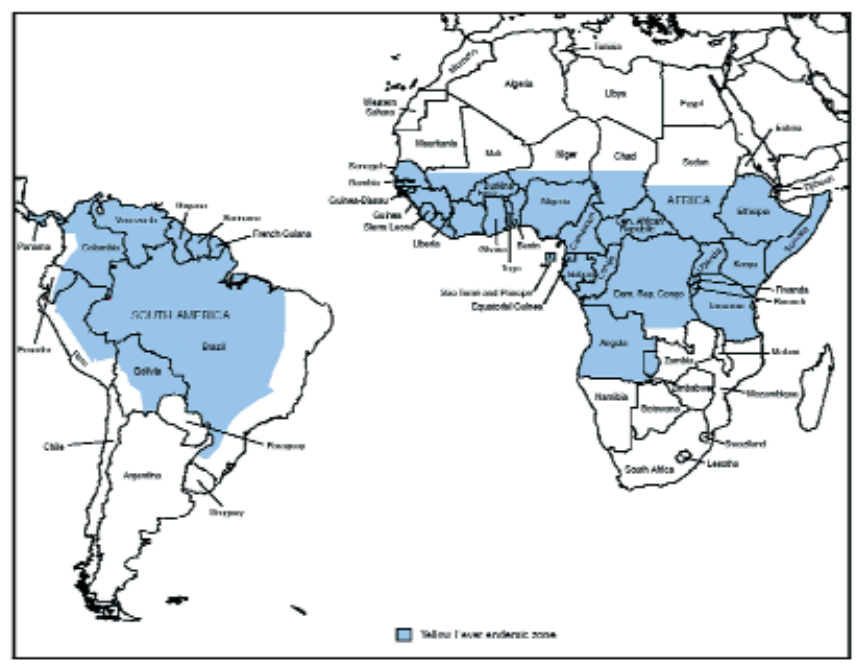

Figura 1. Areas endémicas para fiebre amarilla. Modificado de MMWR 2002, Vol 51 (RR-17). 
géneros como el Alouatta (mono aullador) y el Ateles (mono araña), sucumben en grandes números y marcan con sus cadáveres el paso de la onda epizoótica.

\section{Fisiopatología}

La fisiopatología de la fiebre amarilla no es bien conocida, la mayoría de los estudios realizados han sido en monos Rhesus, los cuales tienen una evolución similar a la de los seres humanos pero con algunas diferencias significativas ${ }^{14}$. Se sabe que el virus de la fiebre amarilla es vicerotrópico, pero en pacientes muy jóvenes puede tener cierto neurotropismo ${ }^{5}$.

A las 24 horas de la inoculación ya han sido infectadas las células de Kupffer, pero éstas todavía se encuentran en buen estado. Entre las 24 y 48 horas las reservas de glicógeno de los hepatocitos comienzan a disminuir, entre las 72 a 96 horas aparecen los primeros cambios en los hepatocitos, evidenciando una migración del virus a partir de las células de Kupffer. La lesión a nivel hepático se localiza en la zona lobulillar media y se produce mediante un mecanismo de apoptosis por degeneración eosinofílica que se evidencia a la microscopía por los cuerpos de Councilman, por la escasa presencia de células inflamatorias en el tejido y por la cicatrización sin fibrosis ${ }^{5,15}$.

Alrededor de las 96 horas el virus alcanza la máxima concentración en sangre, además al examen microscópico ya es posible ver los cuerpos de Councilman, las zonas de necrosis anteriormente focales empiezan a confluir originando la clásica distribución medio zonal, y ya el glicógeno hepático ha desaparecido del todo.

Entre las 96 y las 120 horas la reacción de apoptosis alcanza su plenitud, pero también para ésta etapa ya es posible observar signos precoces de regeneración hepática. A partir de las 96 horas los niveles del virus empiezan a disminuir hasta que llegan a tal punto que a las 120 horas no es posible aislar el virus de la sangre de la mayoría de los pacientes. (Cuadro 1)

La infección del hígado es seguida por la infección de los riñones, el bazo y los nódulos linfáticos. A nivel renal, los riñones se tornan edematosos y la lesión se caracteriza también por una degeneración eosinofílica en el epitelio tubular, con una ausencia casi total de células inflamatorias ${ }^{5,14}$. La oliguria que se presenta es de origen pre-renal debido a la hipotensión asociada y la necrosis tubular aguda, cuando ocurre, es un evento terminal. El resto de los órganos muestran lesiones hemorrágicas focales y congestión difusa ${ }^{14}$.

La hipotensión y el choque en las fases tardías de la enfermedad todavía son motivo de discusión, ya que el
Cuadro 1. Cronograma de la infección

0 Hrs Inoculación

24 Hrs Infección de las células de Kupffer

24-48 Hrs Disminuyen reservas de glicógeno hepático

72-96 Hrs Migración del virus a los hepatocitos

$96 \mathrm{Hrs}$ Viremia máxima

96-120 Hrs Cumbre de la apoptosis hepática, inicia aclaración de la viremia

La infección del hígado es seguida por la infección del bazo, los riñones y los ganglios linfáticos.

corazón se agranda y pierde su tono, indicando una miocardiopatía por lesión viral directa, que es parcialmente responsable por la bradicardia y la hipotensión, pero probablemente estas últimas también sean mediadas por una alteración en la regulación de las citoquinas, otros factores contribuyentes son la acidosis metabólica que se desarrolla simultáneamente ${ }^{14}$.

En cuanto al vómito negro (broza de café), el sangrado gástrico proviene de pequeñas erosiones superficiales de la mucosa gástrica, más que de una lesión mayor ${ }^{4}$.

\section{Cuadro clínico}

El $80 \%$ de las infecciones son sintomáticas, la enfermedad tiene un período de incubación de 3-6 días. Tiene una mortalidad que varía entre $20-50 \%$ según la epidemia. En los casos fulminantes la muerte puede sobrevenir al tercercuarto día, pero comúnmente ocurre al sexto o sétimo.

La enfermedad tiene un inicio súbito, con fiebre alta $\left(38^{\circ}-39^{\circ}\right)$, escalofríos, malestar general, cefalea, fotofobia y mialgias ${ }^{4}$. Durante esta etapa que dura de tres a cuatro días es imposible distinguir la fiebre amarilla de otras enfermedades que ocurren en los trópicos, como el dengue. La fiebre es continua y persiste por 3-4 días antes de mermar ${ }^{16}$, pero puede mantenerse hasta por 11 días. En esta fase de la enfermedad el pulso es muy lento en relación con la temperatura (Signo de Faget) ${ }^{5}$. En los primeros tres días hay abundantes vómitos, pero posterior al tercer día generalmente solo persisten las náuseas, en los pacientes que persisten con vómitos es frecuente que presenten vómitos hemorrágicos debido a numerosas erosiones, pero sin úlceras ${ }^{3,4}$.

Posterior a un breve período de mejoría conocido como el "período de remisión", un 15-25\% de los pacientes presenta nuevamente la clínica durante una segunda etapa de la 
enfermedad llamado "período de intoxicación", en la que se presentan los síntomas de insuficiencia hepática y renal, con tendencia hemorrágica. Un $20-50 \%$ de los pacientes que llegan a padecer compromiso hepatorrenal fallece alrededor de los 7-10 días del inicio de la enfermedad. (Cuadro 2)

La presión arterial generalmente se mantiene estable durante el curso de la enfermedad, notándose un franco descenso en la fase terminal de los casos graves ${ }^{14}$. Cuando la temperatura se mantiene alta por encima de $39^{\circ}$ durante varios días, constituye un signo de mal pronóstico así mismo cuando desciende a la hipotermia ${ }^{4}$. En la etapa terminal de la enfermedad, debido a la alteración de los mecanismos de glicogenolisis y gluconeogénesis se produce hipoglicemia, la cual junto con la hipotensión contribuyen a la acidosis metabólica ${ }^{15}$. La alteración en el nivel de consciencia no es el resultado de una lesión viral directa, más bien es consecuencia de las diversas alteraciones metabólicas.

Al examen físico hay eritema facial, congestión conjuntival, punteado hemorrágico en el velo del paladar, y puede haber dolor epigástrico a la palpación y/o hepatomegalia dolorosa ${ }^{4}$.

La ictericia se observa generalmente del cuarto día de evolución en adelante, siendo más evidente en las palmas, los antebrazos y en la cara anterior del tórax. La melena se ve asociada generalmente a la diarrea y a los vómitos hemorrágicos.

En el hemograma es característica la leucopenia con eosinopenia y linfopenia absolutas, éstas se observan más acentuadas en los casos graves, en los casos benignos va desapareciendo rápidamente en los primeros cuatro días ${ }^{4,14}$.

Las transaminasas se elevan entre las 48 a 96 horas; durante el "período de intoxicación", se profundiza la ictericia y aumentan todavía más las transaminasas con un predominio de aspartato-amino transferasa; el grado de elevación de las transaminasas guarda cierta relación con la intensidad de la enfermedad, particularmente la aspartatoamino transferasa ${ }^{5,14,15}$.

Además se encuentra trombocitopenia, y una prolongación de los tiempos de sangrado y tiempos de protrombina; disminución de los niveles de fibrinógeno y de los factores II, V, VII; VIII, IX y X, también es posible detectar la presencia de productos de degradación de la fibrina ${ }^{5,15}$. Lo anterior sugiere una etiología mixta para el problema de sangrado, por un lado una disminución de la síntesis de los factores de la coagulación debido a la lesión hepática y por el otro una coagulopatía de consumo.

Es posible encontrar anormalidades de la onda S-T en el electrocardiograma, que es la manifestación de la lesión miocárdica ${ }^{3,5}$. (Cuadro 3)

\begin{tabular}{|c|c|}
\hline \multicolumn{2}{|c|}{ Cuadro 2. Clínica de la Fiebre Amarilla } \\
\hline $\begin{array}{ll}\text { - } & \text { Fiebre } \\
\text { - } & \text { Escalofríos } \\
\text { - } & \text { Malestar } \\
\text { - } & \text { Cefalea }\end{array}$ & $\begin{array}{ll}\text { - } & \text { Fotofobia } \\
\text { - } & \text { Mialgia } \\
\text { - } & \text { Nauseas } \\
\text { - } & \text { Vómitos }\end{array}$ \\
\hline \multicolumn{2}{|c|}{ "Periodo de Intoxicación" } \\
\hline $\begin{array}{ll}\text { - } & \text { Hipotensión } \\
\text { - } & \text { Ictericia }\end{array}$ & $\begin{array}{ll}\text { - } & \text { Oliguria } \\
\text { - } & \text { Alteración de la } \\
\text { consciencia }\end{array}$ \\
\hline \multicolumn{2}{|c|}{ Cuadro 3. Alteraciones de Laboratorio } \\
\hline $\begin{array}{ll}\text { - } & \text { Leucopen } \\
\text { - } & \text { Elevación } \\
\text { - } & \text { Alteración } \\
\text { - } & \text { Azoemia } \\
\text { - } & \text { Proteinuri } \\
\text { - } & \text { Hipoglice }\end{array}$ & $\begin{array}{l}\text { aminasas } \\
\text { empos de coagulación }\end{array}$ \\
\hline
\end{tabular}

En cuanto al examen general de orina, es muy frecuente la presencia de albuminuria, debido a la lesión de los túbulos renales, pero ésta disminuye gradualmente, la persistencia prolongada de ésta ultima o el hallazgo de eritrocitos en la orina es de mal pronóstico.

La convalecencia se caracteriza por una prolongada astenia y debilidad. La fiebre amarilla tiende a evolucionar hacia una auto-recuperación espontánea. No hay complicaciones crónicas en riñón, hígado o corazón.

\section{Diagnóstico}

El diagnóstico basado solo en la clínica es prácticamente imposible durante el período de infección así como en formas leves de la enfermedad inclusive durante epidemias. El diagnóstico se confirma de forma definitiva con la serología, al demostrar una elevación de al menos cuatro veces de los títulos de anticuerpos IgM específicos ${ }^{17,18}$. Los anticuerpos $\operatorname{IgM}$ aparecen rápidamente en la primera semana de la enfermedad y alcanzan los niveles máximos en la segunda semana, para luego descender paulatinamente durante los meses siguientes ${ }^{3,10}$.

Cuando se intenta el aislamiento del virus, la muestra de sangre debe tomarse lo antes posible en el curso de la enfermedad, con preferencia antes del quinto día y enviar la muestra en refrigeración. Difícilmente se puede aislar el virus después del cuarto día ${ }^{14}$. 
Para el estudio serológico, se recogerán dos muestras de suero, la primera tomada al principio de la enfermedad y la segunda, dos o tres semanas después; ambas deben enviarse en refrigeración.

En el país no se realiza el examen de serología por parte del Instituto Costarricense de Investigación y Enseñanza en Nutrición y Salud, pero los casos sospechosos son enviados a Panamá para su respectivo análisis. En Panamá se realiza reacción en cadena de la polimerasa buscando ARN viral para aquellos pacientes con menos de 4 días de evolución y un ELISA (IgM) para aquellos con más de 4 días. Desde enero a octubre del 2004 se enviaron desde Costa Rica tres muestras a Panamá buscando fiebre amarilla (resultado negativo en las tres).

Hay un problema de reacción cruzada a la hora de realizar el ELISA en pacientes que han tenido dengue (o alguna otra infección por flavivirus) que puede resultar en falsos positivos ${ }^{3,5,7,19}$ por lo que en esos casos se recomienda realizar un Western-blot, otra opción que esta disponible es la detección del ARN viral ${ }^{20}$. Debido a la naturaleza del diagnóstico serológico (es retrospectivo), es de mayor valor epidemiológico que clínico.

El diagnóstico también se puede establecer por biopsia hepática post mortem ${ }^{3,10}$, pero esta no siempre es diagnósti$\mathrm{ca}^{14}$; nunca se debe tratar de obtener una biopsia hepática en un paciente vivo con fiebre amarilla debido al riesgo de sangrado masivo ${ }^{10}$.

\section{Diagnóstico diferencial}

\section{Malaria}

La clínica inicial es inespecífica, con malestar general, cefalea, fatiga, malestar abdominal y fiebre, no hay rigidez nucal o fotofobia pero si vómitos y náuseas. El patrón paroxístico de la fiebre es característico y se acompaña de taquicardia; en ocasiones puede haber ictericia leve. En los exámenes de laboratorio se verá una leve anemia normocrómica-normocítica, trombocitopenia, con leucocitos normales o disminuidos y un examen de orina normal. El diagnóstico se confirma al demostrar el parásito mediante el frotis sanguíneo, la serología no es muy útil.

\section{Tifoidea}

Tiene un período de incubación de 10-14 días, se caracteriza por fiebre, escalofríos, malestar general, en el $90 \%$ de los casos aparece un rash macular en el tronco, puede haber insuficiencia renal y delirios. En el examen general de orina se puede encontrar proteinuria y hematuria. El diagnóstico se confirma con la serología y tiene un período de recuperación de 13 a 16 días ${ }^{16}$.

\section{Dengue}

Tiene un período de incubación de 2 a 7 días, el paciente experimenta fiebre de comienzo brusco, cefalea, dolor retro-orbitario y dolores de espalda, además de mialgias intensas. En el primer día suele haber un exantema maculo-papuloso, así como adenopatías, vesículas en el paladar e inyección conjuntival. La enfermedad puede durar una semana y existen habitualmente otros síntomas como anorexia, náuseas o vómitos, e intensa hipersensibilidad cutánea $^{21,22}$.

Los hallazgos de laboratorio consisten en leucopenia, trombocitopenia y en muchos casos, elevación de las transaminasas. El diagnóstico realizado mediante ELISA de IgM durante la recuperación o mediante reacción en cadena de la polimerasa para ARN viral durante la fase aguda.

\section{Leptospirosis}

Tiene un período de incubación de 2-20 días. Hay dos variantes clínicas de la enfermedad, la forma anictérica es la más común, ésta tiene una fase "séptica" con un inicio súbito de fiebre, dolor abdominal, cefaleas y mialgias; luego de un período de 1-3 días de mejoría inicia la fase "inmune" con una recaída de los síntomas que puede asociarse a meningitis. Es autolimitada y dura de 4-30 días. El síndrome de Weil es la forma severa de la enfermedad, con alteraciones de la función renal y hepática, alteración del estado mental e hipotensión. La clínica es continua, no bifásica como en la forma leve ${ }^{23}$.

En los exámenes de laboratorio generalmente se encuentra leucocitosis, elevación de la creatinina kinasa, en la orina pueden encontrarse proteínas y eritrocitos. Aunque el organismo puede ser visto mediante un campo oscuro, el diagnóstico se confirma con la serología.

\section{Hepatitis B}

Luego de la infección por hepatitis B hay de un período de incubación que va desde 40 a 180 días, posterior al cual se presenta la fase pre-ictérica de la enfermedad que dura de 5 a 15 días, caracterizada por síntomas de tipo gripal con febrícula, artralgias, astenia, anorexia, plenitud, náuseas y a veces vómito y cefalea. La fase ictérica es de intensidad variable, dura de 2 a 8 semanas y va precedida de 2 a 3 días de coluria, que puede complementarse con hipocolia o acolia. En este período el paciente pese a estar ictérico, mejora notablemente de sus síntomas prodrómicos ${ }^{16,24}$.

El cuadro típico cursa con leucopenia y linfocitosis relativa, un aumento de transaminasas que puede ser hasta 10 veces más de lo normal, hay hiperbilirrubinemia de predominio directo y prolongación de los tiempos de coagulación. El diagnóstico se confirma con la serología, que es útil para diferenciar los diferentes estadíos de la enfermedad. 


\section{Tratamiento}

No hay un tratamiento específico, el manejo es principalmente de soporte; hace algunos años se recomendó el siguiente manejo: mantener la nutrición y prevenir la hipoglucemia, aspirado gástrico para prevenir la distensión, cimetidina intravenosa para prevenir el sangrado gástrico, tratamiento de la hipotensión mediante la restitución de fluidos y drogas vasopresoras (dopamina), administración de oxígeno, corrección de la acidosis metabólica, tratamiento del sangrado con plasma fresco congelado y diálisis según sea necesario por el grado de falla renal y tratamiento con antibióticos para las infecciones secundarias (en particular la neumonía) ${ }^{5,15}$. Estas recomendaciones establecidas por sentido común no han sido modificadas desde que fueron establecidas, pero tampoco han sido corroboradas por ningún estudio.

Con base en su gran mortalidad y a la ausencia de un tratamiento específico es obvio que el camino a seguir en el manejo de la fiebre amarilla es la prevención mediante las vacunaciones masivas en las áreas endémicas y grupos de riesgo, así como el control del vector.

\section{Prevención}

Para la prevención de la fiebre amarilla se cuenta con una vacuna, la cepa D17 ${ }^{25}$, la cual fue concebida por Max Theiler en 1936, es una vacuna viva atenuada incubada en huevos de pollo.

Estimula niveles protectores de anticuerpos a los 10 días de la inoculación en alrededor de $86-88 \%$ de los pacientes (es a partir de los 10 días que el sujeto se considera como protegido) y en el $98-99 \%$ a los treinta días y aunque se estima que esta protección es de por vida se recomienda un refuerzo cada 10 años ${ }^{5,26,27}$. Las reacciones adversas mas frecuentes son cefalea, mialgia, febrícula u otros síntomas leves.

No se recomienda en niños menores de 9 meses por el riesgo de encefalitis ${ }^{28,29}$ también conocida como "enfermedad neurotrópica asociada a la vacuna de fiebre amarilla", otras contraindicaciones son la inmunosupresión (en el caso de los pacientes VIH positivos, si su conteo de CD4 es mayor de 200, estos pueden ser vacunados pero la respuesta inmunogenica puede no ser lo suficientemente adecuada para brindar protección ${ }^{30}$ ), el embarazo y la alergia a los componentes de la vacuna, en particular al huevo. Se recomienda la vacunación de todas las personas que vivan en áreas endémicas o que vayan a viajar a las mismas, que no presenten ninguna de las contraindicaciones ya citadas ${ }^{31,32}$.

La vacuna D17 era considerada una de las vacunas más seguras que existía pero recientemente han salido a la luz reportes de reacciones adversas fatales post-vacunación ${ }^{33-35}$. Los estudios de varios casos de este tipo de reacciones ocurridas en Estados Unidos, Australia y Brasil no han llegado a una explicación concreta del porque de éstas reacciones. Las teorías propuestas mencionan la posibilidad de una respuesta anormal del hospedero en algunos de los casos y en los otros se habla de que sub-poblaciones del virus D17 que están en la vacuna o mutaciones de novo en el ARN viral que aparecen en el hospedero, sufren una ampliación selectiva la cual es capaz de causar una enfermedad clínicamente significativa ${ }^{36}$ conocida como "enfermedad viscerotrópica asociada a la vacuna de la fiebre amarilla".

Casi todos los países endémicos para fiebre amarilla de América del Sur exigen la tarjeta de vacunación para viajeros procedentes de países endémicos o con brotes activos de la enfermedad ${ }^{37} \mathrm{y}$ aunque esta normativa se encuentra incluida en el reglamento sanitario internacional ${ }^{38}$, en nuestro país hasta el momento no se solicita este comprobante a los extranjeros que vienen de países endémicos. (Cuadro 4)

Según recomendaciones de la Organización Mundial de la Salud, los países en riesgo de epidemias de fiebre amarilla deberían tener almacenadas una reservada de vacunas contra la fiebre amarilla ${ }^{39}$, y aunque a nivel mundial son varios los laboratorios que fabrican la vacuna, a nivel nacional solo se puede conseguir de forma privada.

\section{Conclusión}

La fiebre amarilla es una patología que algunos no han oído nombrar y que muchos consideran una enfermedad de tiempos pasados, pero en realidad si bien su incidencia se ha visto reducida en relación con las epidemias de antaño, sigue siendo una entidad que afecta considerablemente la salud pública en varios países del mundo. Costa Rica a pesar de no tener casos reportados, si reúne un conjunto de condiciones que facilitaría su aparición y propagación, por lo que es una patología cuyas características convendría mantener a la mano, además hay que tener en cuenta que las medidas preventivas dirigidas hacia la fiebre amarilla son relativamente fáciles de implementar y de gran costoefectividad. 


\begin{tabular}{|lcc|}
\hline Cuadro 4. Situación del Continente Americano \\
\hline Países & $\begin{array}{c}\text { Países } \\
\text { endémicos }\end{array}$ & $\begin{array}{c}\text { Requiere vacunación } \\
\text { para turistas } \\
\text { provenientes de países } \\
\text { endémicos }\end{array}$ \\
\hline Belice & $\mathbf{x}$ & $\mathbf{x}$ \\
Bolivia & $\mathbf{x}$ & $\mathbf{x}$ \\
Brasil & $\mathbf{x}$ & $\mathbf{x}$ \\
Colombia & & $\mathbf{x}$ \\
Costa Rica & $\mathbf{x}$ & \\
Ecuador & & $\mathbf{x}$ \\
El Salvador & & $\mathbf{x}$ \\
Guatemala & $\mathbf{x}$ & $\mathbf{x}$ \\
Guyana Francesa & $\mathbf{x}$ \\
Honduras & & $\mathbf{x}$ \\
Nicaragua & & $\mathbf{x}$ \\
Panamá & $\mathbf{x}$ & $\mathbf{x}$ \\
Paraguay & & $\mathbf{x}$ \\
Perú & $\mathbf{x}$ & $\mathbf{x}$ \\
Venezuela & & \\
\hline Modificado de: www.who.int/ith/Chapter05_ & & \\
\hline & & \\
\hline
\end{tabular}

\section{Agradecimientos}

Queremos agradecer la ayuda brindada por el Dr. Jaime Solís Q del Hospital San Juan de Dios y al Dr. Willy Carrillo A. de la Dirección de Vigilancia de la Salud, del Ministerio de Salud.

\section{Abstract}

Yellow fever is an acute febrile zoonosis, catalogued as a hemorrhagic fever that is potentially deadly. It is caused by an arbovirus and transmitted by an arthropod; there are two known epidemiologic modalities, and it has an endemic area that comprises most of South America and part of the African continent. In Costa Rica the last outbreak of yellow fever was in the fifties.

The physiopathology of yellow fever is not well known. Most of the infections are symptomatic and have a high mortality that varies from outbreak to outbreak. The diagnosis is definitely confirmed by serology and although this test isn't performed in Costa Rica, the suspicious samples are sent to Panamanian labs.

Taking into consideration its high mortality and the lack of specific treatment, it's obvious that the only way to deal with yellow fever is to get rid of the vector and prevention with massive vaccination in endemic areas.

Yellow fever continues to be an entity that considerably affects public health in several countries; Costa Rica has quite a few conditions that will ease its appearance and dissemination, that's why this is a disease which's characteristics are convenient to keep handy.

\section{Referencias}

1. Renan A. La fiebre amarilla en Yucatán durante las épocas precolombina y colonial. Biomédica 2000; 11: 301-307.

2. Mc Carthy, M. A century of the US army yellow fever research. Lancet 2001; 357: 1772.

3. Strano A, Dooley J, Isaac K. Manual sobre la fiebre amarilla y su diagnostico diferencial histopatológico. Publicación científica de la OPS No. 299, 1975

4. Trejos A, Romero A. Clínica de la fiebre amarilla en Costa Rica. Rev Biol Trop 1954; 113-168.

5. Monath T. Yellow fever: an update. Lancet Infect Dis 2001; 1: 11-20.

6. Mutebi J, Wang H, Li L, Bryant J., and Barrett A. Phylogenetic and evolutionary relationships among yellow fever virus isolates in Africa. J Virol 2001; 6999-7008.

7. Gubler D. Aedes albopticus in Africa. Lancet Infect Dis 2003; 13: 751.

8. Van der Stuyft P, Gianella A, Pirard M, Cespedes J, Lora J, Peredo C, et al. Urbanization of yellow fever in Santa Cruz, Bolivia. Lancet 1999; 353: 1558-1562.

9. Monath T. Facing up to re-emergence of urban yellow fever. Lancet 1999; 353: 1541 .

10. OPS. Guías para la vigilancia, prevención y control de la fiebre amarilla. Publicación Científica no. 410 Organización Panamericana de la Salud 1981

11. Velandia M. Fiebre Amarilla Selvática, Colombia 2003. Publicación del Instituto Nacional de Salud 2003.

12. Reiter P, Cordellier R, Ouma J, Cropp B, Savage H, Sanders E, et al. First recorded outbreak of yellow fever in Kenya 1992-1993. Am. J Trop Med Hyg, 1998; 59: 650-656.

13. Thonnon J, Fontenille D, Tall A, Diallo M, Renaudinean Y, Baudez B, et al. Re-emergence of yellow fever in Senegal in 1995. Am. J Trop Med Hyg 1998; 59: 108-114.

14. Monath T, Brinker K, Chandler F, Kemp G, Cropp B. Pathophysiologic correlations in a rhesus monkey model of yellow fever. Am J Trop Med Hyg 1981; 30: 431-41.

15. Monath T. Yellow fever: a medically neglected disease. Report on a seminar. Rev Infect Dis 1987; 9: 165-175.

16. Fauci A, Braunwald E, Isselbacher K, Wilson J. Harrison, Principios de Medicina Interna. Estados Unidos: Editorial Mc Graw-Hill Internacional, 1998. 
17. WHO. Epidemiological bulletin, March 1999; 1: 20.

18. Vasconcelos P. Diagnosis of viral disease. Lancet 2003; 361: 1589.

19. Campbell G, Marfin A, Lanciotti R, and Gubler D. West Nile virus. Lancet Infect Dis 2002; 2: 519-529.

20. Monath T, Ballinger M, Miller B, Salaun J. Detection of yellow fever viral RNA by nucleic acid hybridization and viral antigen by immunocytochemistry in fixed human liver tissues. Am J Trop Med Hyg 1989; 40: 663-68.

21. Gubler, D. Dengue and dengue hemorrhagic fever. Clin Microbiol Rev 1998; 11: 480-496.

22. Guzmán M, Kourí G. Dengue: an update. Lancet Infect Dis 2002; 2: 33-42.

23. Tierney L, McPhee S, Papadakis M. Current Medical Diagnosis \& Treatment 2002. United States, Lange Medical Books/ McGraw-Hill 2002.

24. Berhman R, Kliegman R, Jenson H. Nelson, Tratado de Pediatría. México, Estados Unidos: Editorial McGraw-Hill Interamericana, 2001.

25. Mortimer P. Yellow fever vaccine. BMJ 2002; 324-23; 439.

26. Monath T, Nichols R, Archambault W, Moore L, Marchesani R, Tian J, et al. Comparative safety and immunogenicity of two yellow fever $17 \mathrm{~d}$ vaccines (arilvax and yf-vax) in a phase III multicenter, doubleblind clinical trial. Am. J Trop Med Hyg 2002; 66: 533-541.

27. Lang J, Zuckerman J, Clarke P, Barrett P, Kirkpatrick C, and Blondeau C. Comparison of the immunogenicity and safety of two 17d yellow fever vaccines. Am. J Trop Med Hyg 1999; 60: 1045-1050.

28. Peter G, Hall C, Halsey N, Marcy S, Pickering L. Red Book, Enfermedades Infecciosas en Pediatría. Argentina: Editorial Medica Panamericana, 1999.

29. CDC. Yellow Fever Vaccine: Recommendations of the Advisory Committee on Immunizations Practices. MMWR 2002, vol 51 (RR17).
30. Sibailly T, Wiktor S, Tsai T, Cropp B, Ekpini E, Adjorlolo-Johnson G, et al. Poor antibody response to yellow fever vaccination in children infected with human immunodeficiency virus type 1. Pediatr Infect Dis J 1997; 16: 1177

31. Robert E, Vial T, Schaefer C, Arnon J, Reuvers M. Exposure to yellow fever vaccine in early pregnancy. Vaccine 1999; 17: 283-85.

32. Tsai T, Raul R, Lynberg M, Letson G. Congenital yellow fever virus infection after immunization in pregnancy. J Infect Dis. 1993; 168: 1520 .

33. Martin M, Tsai T, Cropp B, Chang G, Holmes D, Tseng J, et al. Fever and multisystem organ failure associated with 17d-204 yellow fever vaccination: a report of four cases. Lancet 2001; 358: 98-104.

34. Chan R, Penney D, Little D, Carter I, Roberts J, Rawlinson W. Hepatitis and death following vaccination with 17d-204 yellow fever vaccine. Lancet 2001; 358: 121-122.

35. Vasconcelos P, Luna E, Galler R, Silva L, Coimbra T, Barros V, et al. Serious adverse events associated with yellow fever $17 \mathrm{dd}$ vaccine Brazil: a report of two cases. Lancet 2001; 358: 91-97.

36. Jennings A, Gibson C, Miller B, Mathews J, Mitchell C, Roehrig J, et al, . Analysis of a yellow fever virus isolated from a fatal case of vaccine-associated human encephalitis. J Infect Dis 1994; 169: 512-18.

37. WHO, International Travel and Heath. Switzerland: WHO 2005.

38. WHO, International Heath Regulations (1969): third annotated edition, Geneva, World Health Organization, 1983.

39. PAHO, Conclusions and recommendations of the technical advisory group on vaccine preventable diseases, Municipalities: Improving Immunization Services, of the Pan American Health Organization 2002. United States: PAHO 2002. 